\title{
Editorial
}

\section{Innovative Nanomaterials for Thermal Applications}

\author{
Yan Wang, ${ }^{1}$ Hua Bao, ${ }^{2}$ Yalin Dong, ${ }^{3}$ Rajib Paul, ${ }^{4,5}$ and Hua Deng ${ }^{6}$ \\ ${ }^{1}$ Department of Mechanical Engineering, University of Nevada, Reno, NV 89557, USA \\ ${ }^{2}$ University of Michigan-Shanghai Jiao Tong University Joint Institute, Shanghai Jiao Tong University, Shanghai 200240, China \\ ${ }^{3}$ Department of Mechanical Engineering, University of Akron, Akron, OH 44325, USA \\ ${ }^{4}$ Department of Macromolecular Science and Engineering, Case Western Reserve University, Cleveland, OH 44106, USA \\ ${ }^{5}$ Department of Physics, Heritage Institute of Technology, Anandapur, Kolkata 700107, India \\ ${ }^{6}$ Western Digital Corporation, San Jose, CA 95131, USA \\ Correspondence should be addressed to Yan Wang; wang896@purdue.edu
}

Received 6 November 2016; Accepted 7 November 2016; Published 29 January 2017

Copyright (c) 2017 Yan Wang et al. This is an open access article distributed under the Creative Commons Attribution License, which permits unrestricted use, distribution, and reproduction in any medium, provided the original work is properly cited.

With continued advancement in nanotechnology, numerous innovative nanomaterials, possessing internal features or surface structures of nanoscale dimension, have emerged as golden materials for sustainable and efficient energy solutions. Thermal properties of nanomaterials depend upon many factors that are usually insignificant in bulk materials. In particular, surface properties, interfacial structures, and quantum or classical size effects prominently determine the thermal transport in nanomaterials, leading to carrier scatterings and localization that are otherwise absent or unobvious in bulk materials.

Through this special issue, we aim to enlighten the fundamental understanding of thermal transport and energy conversion processes at the nanoscale and facilitate the development of innovative nanomaterials for thermal applications.

In $\mathrm{D}$. Zhao et al's work, nanosized $\mathrm{C}_{60}$ powder was incorporated with $\mathrm{Cu}_{2} \mathrm{GeSe}_{3}$ powder by ball milling and then $\mathrm{C}_{60} / \mathrm{Cu}_{2} \mathrm{GeSe}_{3}$ composites were prepared by spark plasma sintering. The authors found that as the concentration of $\mathrm{C}_{60}$ increases, the electrical resistivity and the Seebeck coefficient of $\mathrm{C}_{60} / \mathrm{Cu}_{2} \mathrm{GeSe}_{3}$ composites increase while the thermal conductivity decreases significantly. A maximum figure of merit ZT of 0.20 has been achieved at $700 \mathrm{~K}$ for the $0.9 \%-\mathrm{C}_{60} / \mathrm{Cu}_{2} \mathrm{GeSe}_{3}$ composites, rendering this material potentially useful for high temperature applications.

In P. Zhang et al.'s work, low-temperature sintered nanosilver pastes were attempted to interconnect large-power LED and substrates. The authors found that the shear strength of the low-temperature sintered nanosilver paste increases as the temperature or pressure increases. This material also shows relatively low thermal resistance.

$\mathrm{X}$. Zhang and G. Wu conducted nonequilibrium molecular dynamics simulations of strained Si thin films. The thermal conductivity was found to decrease as the tensile strain increases and increase as the compressive strain increases, which could aid the modification of the thermal conductivity, and, moreover, the thermal mangement of Si-thin-film-based devices.

Z. Chai et al. investigated nonlinear absorption of CdSeTe quantum dots in toluene using the femtosecond Z-scan technique at $800 \mathrm{~nm}$. The authors investigated the openaperture Z-scan behaviors of the samples on the local nonlinear responses, including two-phonon absorption (TPA) coefficient. It was demonstrated that, under the conditions of high-repetition rate, heat accumulation and bleaching of ground state are responsible for the decrease of the TPA coefficient.

J. Wang et al. proposed a multiple plasmon-induced transparency (PIT) device operated in the midinfrared region and investigated the phase coupling between two graphene resonators. The multimode PIT resonances were found to be dynamically tunable by varying the chemical potential of graphene resonators, which could be potentially useful for various energy applications.

$\mathrm{X}$. Huang et al. used the density functional theory to study the chemisorption of $\mathrm{NO}$ and successive heterogeneous 
reduction mechanisms on the well-defined char models under carbon/char- $-\mathrm{CO}_{2}$ gasification condition. Their results indicate that intensive gasification surface is likely to be thermally unfavorable and the O-down mode is regarded as the most inactive pathway for the absorption of NO.

All these papers show how nanomaterials can be well understood and utilized for various thermal and energy applications.

\section{Acknowledgments}

The guest editors would like to thank the authors who contributed to this issue. The lead guest editor would like to thank all the other guest editors for their dedication to this issue.

Yan Wang

Hua Bao

Yalin Dong

Rajib Paul

Hua Deng 

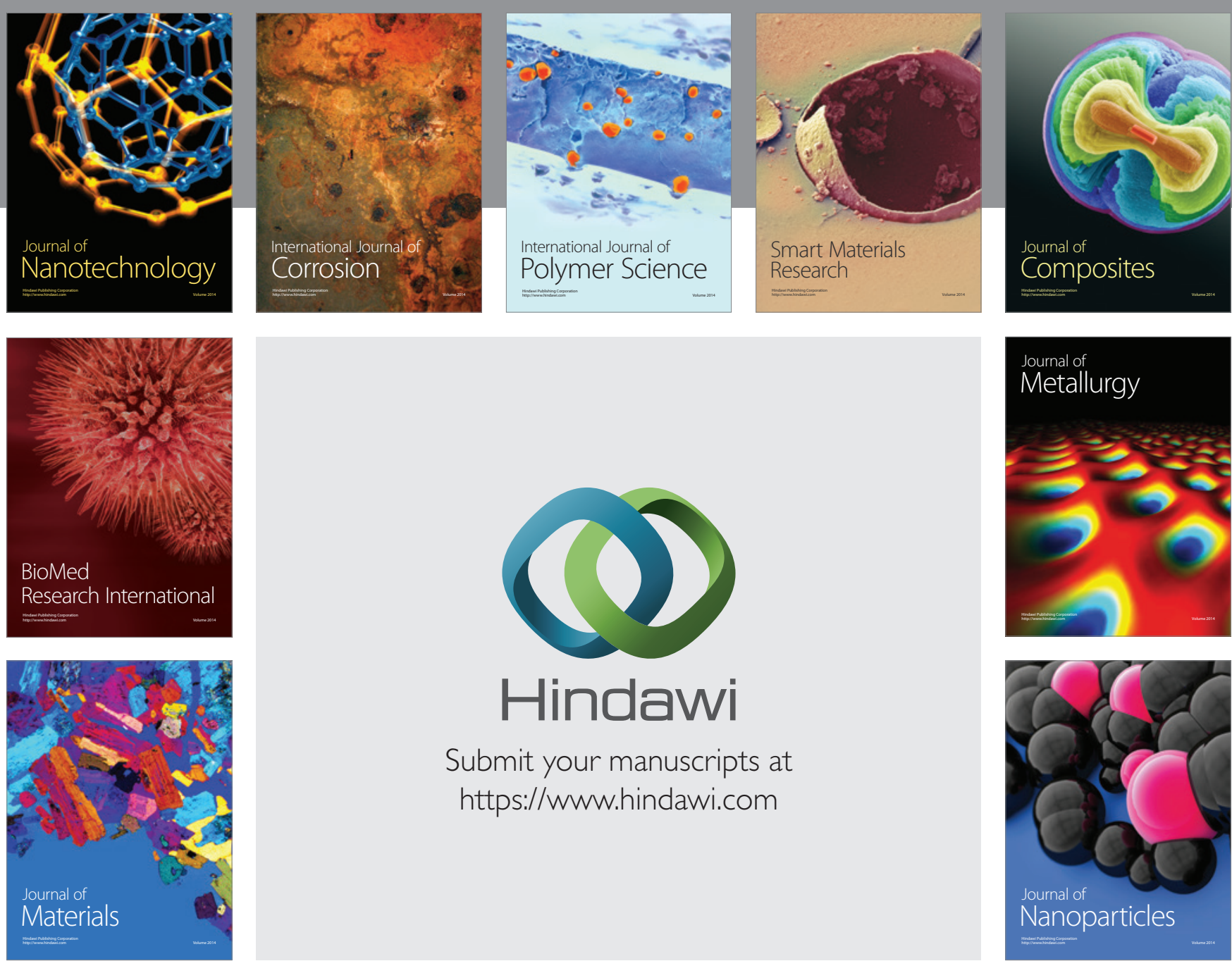

\section{Hindawi}

Submit your manuscripts at

https://www.hindawi.com

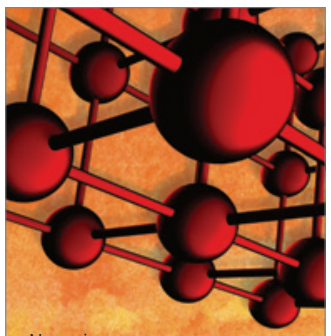

Materials Science and Engineering
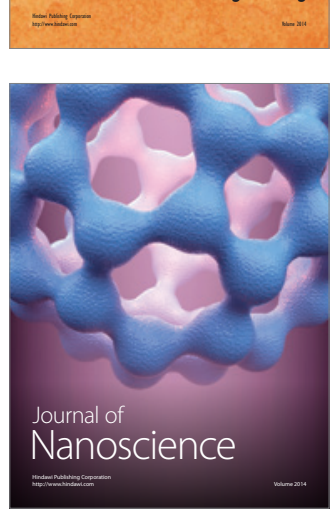
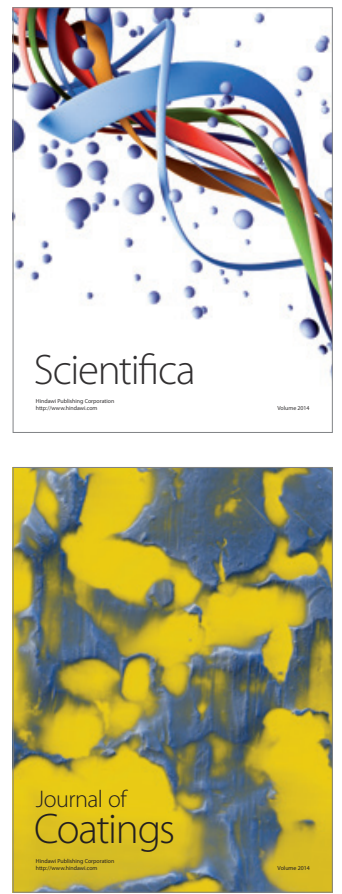
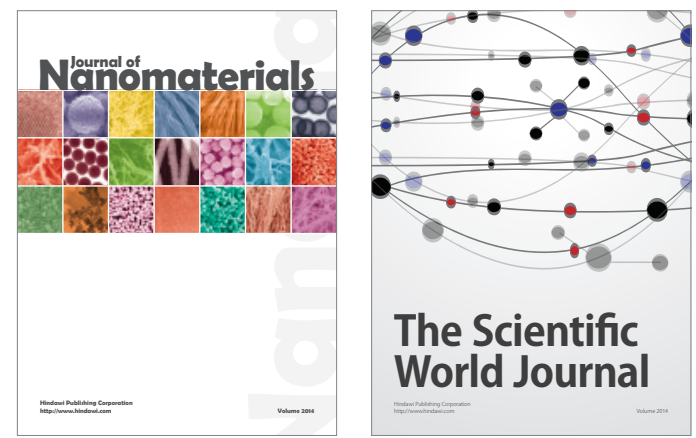

The Scientific World Journal
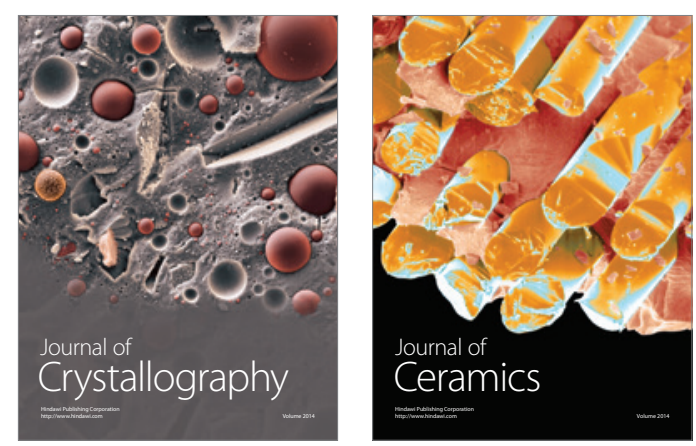
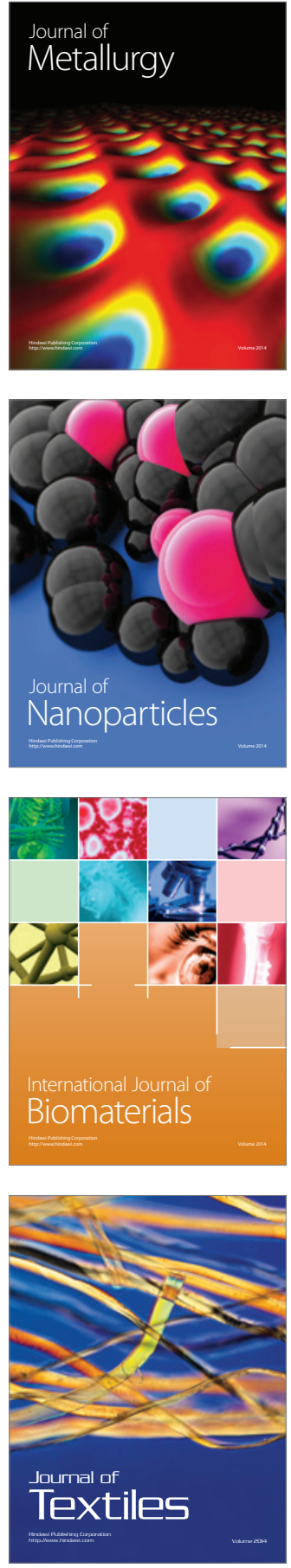